\title{
Death at the Dead Sea
}

\author{
S Bialik ${ }^{1}$ and T Gottlieb ${ }^{2}$ \\ 1 Department of Molecular Genetics, Weizmann Institute of Science, Rehovot, Israel \\ 2 Department of Molecular Cell Biology, Weizmann Institute of Science, Rehovot, Israel
}

The Seventh European Conference on Apoptosis (European Cell Death Organization and European Haematology Association) at Ein Gedi, Israel, 14-17 November, 1999

Abbreviations: $\mathrm{BH} 3, \mathrm{Bcl}-2$ homology domain 3; CAD, caspase activated DNase; CARD, caspase recruitment domain; DAP, death associated protein; DD, death domain; DED, death effector domain; DISC, death-inducing signaling complex; DR, death receptor; elF4, eukaryotic initiation factor 4; FADD, Fas associated death domain protein; FasL, Fas ligand; FLIP, FLICE-inhibitory protein; GFP, green fluorescent protein; Hsp, heat shock protein; IAP, inhibitor of apoptosis; ICAD, inhibitor of caspase activated DNase; IRES, internal ribosome entry site; JNK, jun N-terminal kinase; NGF, nerve growth factor; Nrf2, NF-E2 related factor; PI3K, phosphatidylinositide-3'-OH kinase; PT, permeability transition; RAIDD, RIPassociated Ich-1/CED-3 homologous protein with death domain; $\mathrm{RIP}$, receptor interacting protein; ROS, reactive oxygen species; TKO, technical knockout; TNF $\alpha$, tumor necrosis factor $\alpha$; TNFR, TNF $\alpha$ receptor; TRADD, TNF receptor associated death domain protein; TUNEL, terminal deoxynucleotidyl-mediated dUTP nick end labeling; UTR, untranslated region, WRN, Werner protein

The Seventh European Conference on Apoptosis was held on November 14-17, 1999, in the appropriate setting of Kibbutz Ein Gedi, Israel, overlooking the Dead Sea. The desolation of the Dead Sea was stirred to life by the verdant landscaping of the kibbutz and the varied wildlife in the Ein Gedi oasis, much as the topic of cell death was invigorated by the dynamic speakers present at the meeting. This report will highlight certain themes and novel perspectives presented at the meeting, focusing on (1) the central apoptosis machinery (2) signaling to the death machinery and (3) non-conventional caspase-independent cell death. In instances where the research discussed has been published, the reader is referred to the appropriate reference for further details.

\section{THE CENTRAL APOPTOTIC MACHINERY}

The past decade of research in the field of apoptosis has focused on elucidating the molecular components of the basic machinery that instructs a cell to die. The most prominent component of the central apoptotic machinery is the caspase family of cysteine proteases. Caspases lie in a dormant, inactive state in the normal cell. Apoptotic signals lead to their activation through a cascade of proteolytic cleavage. ${ }^{1}$ This cascade involves auto-activation and processing of the most upstream caspases, the 'initiator' caspases, which then process and activate the 'effector' caspases. These latter caspases, which include caspase-3, proteolyze the specific cell substrates whose cleavage enables the series of biochemical and morphological changes that characterize the apoptotic cell. Additional proteins that serve to activate the caspase cascade are also considered to be part of the apoptotic machinery.

\section{The initiation phase: activation of caspases}

The initiator caspases are activated in the context of the apoptosome, a complex of proteins that facilitates self cleavage by bringing two or more initiator caspases into close proximity. Two types of apoptosome, that assemble in response to particular apoptotic stimuli, have been described. ${ }^{1}$ Formation of one apoptosome is dependent upon mitochondrial release of cytochrome $c$, which complexes with Apaf-1 and caspase-9. The second type forms at the cell membrane in response to death receptor activation, and includes the death receptors, adaptors such as FADD or TRADD, and caspase-8. A link between the two apoptosomes lies in Bid, a BH3-domain-containing pro-apoptotic member of the $\mathrm{Bcl}-2$ family. Bid is cleaved by caspase- 8 following activation of death receptors, enabling it to translocate to the mitochondria and promote cytochrome $c$ release. ${ }^{2,3}$ Both mitochondrial and death receptor mediated activation of caspases were discussed at the meeting, with emphasis on the former pathway, which remains more enigmatic.

\section{Mitochondrial dependent caspase activation}

The Apaf-1/caspase-9 apoptosome is formed following the release of cytochrome $c$ from the intermembrane space of the mitochondria. Much evidence points to Bax, a pro-apoptotic member of the Bcl-2 family, as a mediator of cytochrome $c$ release. Jean-Claude Martinou (Serono Pharmaceutical Research Institute, Geneva, Switzerland) explored the validity of two opposing models that potentially explain the ability of Bax to induce cytochrome $c$ release. The first proposes that Bax forms a specific channel in the outer mitochondrial membrane (model 1), while the second states that Bax activates the permeability transition (PT) pore, causing non-specific cytochrome $c$ leakage from mitochondria following depolarization, swelling and rupture of the outer mitochondrial membrane (model 2). Martinou presented several experimental results that favor the former model. The drug cyclosporine $\mathrm{A}$, which closes the adenine nucleotide 
transporter, a component of the PT pore, does not prevent Bax-mediated release of cytochrome $c$ from isolated mitochondria. Furthermore, mitochondrial swelling and outer membrane damage, characteristics of PT, are not observed, but rather mitochondria appear to shrink. Model 1, in which Bax functions as a specific channel for cytochrome $c$, is consistent also with Martinou's report that in NGF-deprived sympathetic neurons, depletion of cytochrome $c$ from mitochondria is a reversible event. ${ }^{4}$

Douglas Green (La Jolla Institute for Allergy and Immunology, California, USA) described a series of elegant experiments which, in line with model 1, indicate that cytochrome $c$ release is a controlled process that does not require loss of mitochondrial integrity. He used GFPtagged cytochrome $c$ expressed stably in HeLa cells to analyze the kinetics of release in response to actinomycin D, staurosporine, UV irradiation and $\mathrm{TNF}_{\alpha} .^{5}$ In each case, there is a lag time before cytochrome $c$ is released, but once release begins, it is completed by a cell within $5 \mathrm{~min}$, and individual mitochondria can lose all their cytochrome $c$ within as little as $1 \mathrm{~min}$. This occurs hours before other apoptotic events, such as phosphatidylserine exposure, are observed. The ' 5 min' cytochrome $c$ release was seen even in the presence of caspase inhibition (except for $\mathrm{TNF} \alpha$ ) and also at low temperatures. Interestingly, in the presence of caspase inhibitors, mitochondrial respiration continued and membrane polarization was maintained despite the apparent release of cytochrome $c$. Thus, cytochrome $c$ release occurs independently of PT.

According to both models, the ability of Bax to induce release of cytochrome $c$ is modulated by other members of the Bcl-2 family. Martinou presented data regarding the regulation of $\mathrm{Bax}$ by $\mathrm{Bid}$, a $\mathrm{BH} 3$-domain pro-apoptotic member of the Bcl-2 family. ${ }^{6}$ Bid is cleaved by caspase- 8 following activation of death receptors, enabling it to translocate to the mitochondria and promote cytochrome $c$ release. ${ }^{2,3}$ Martinou reported that addition of Bid to Bax in the presence of isolated mitochondria induced a shift in Bax from a monomeric, $24 \mathrm{kDa}$ form to dimeric and trimeric forms of $\sim 40$ and $60 \mathrm{kDa}$. Such oligomerization of Bax is considered important for its pro-apoptotic activity. ${ }^{7}$ Furthermore, Bid caused a change in conformation in Bax, enabling the latter to insert into the mitochondrial membrane. $\mathrm{Bcl}-2 / \mathrm{Bcl}-\mathrm{x}_{\mathrm{L}}$, on the other hand, can block this change in conformation. ${ }^{6}$

There is evidence to suggest that the anti-apoptotic proteins of the Bcl-2 family can promote survival by additional mechanisms. Eyal Gottlieb (University of Pennsylvania, USA) presented data concerning the relationship between the $\mathrm{Bcl}-2$ family and mitochondrial physiology following TNF $\alpha$-induced signal transduction. He examined the mechanism by which $\mathrm{Bcl}-\mathrm{X}_{\mathrm{L}}$ interferes with TNF/cycloheximide- and $\mathrm{H}_{2} \mathrm{O}_{2}$-induced apoptosis. The antiapoptotic properties of $\mathrm{Bcl}-\mathrm{X}_{\mathrm{L}}$ correlated with its ability to inhibit complete mitochondrial depolarization following generation of ROS. Gottlieb proposes that $\mathrm{Bcl}-\mathrm{X}_{\mathrm{L}}$ blocks this ROS generation by mitochondria, thus inhibiting the depolarization cycle, but does not act as a non-specific anti-oxidant. The mechanism by which this is accomplished is not clear.
The release of cytochrome $c$ from the mitochondria facilitates the formation of the Apaf-1/caspase- 9 apoptosome. Seamus Martin (National University of Ireland, County Kildare) examined Apaf-1 dimerization by yeast-2 hybrid analysis. The ced-4 homology domain and the region that links this domain to the WD-40 repeats are the minimal requirements for homo-dimerization. The ability of Apaf-1 deletion constructs to dimerize correlates with their ability to activate caspase-9. The WD-40 domain blocks dimerization, as well as caspase- 9 binding to the CARD domain, unless cytochrome $c$ and dATP are present. Consistent with this, Apaf-1 lacking the WD-40 domain is a more potent killer in MCF-7 cells than the full length protein. ${ }^{8}$ Martin also described the hierarchy of caspase activation following cytochrome $c$ activation of Apaf- 1 in a cell free system. Using GST-pull down experiments, he found that the CARD domain of Apaf-1 bound exclusively to caspase-9, and not to the prodomains of other caspases assayed. Caspase-9, after activation by binding to Apaf-1, processed caspase- 3 and caspase-7, as has been previously reported. ${ }^{9}$ Caspase-3 was then responsible for activating caspase- 2 and caspase-6, which in turn proteolyzed caspase-8 and -10 .

Gerald Cohen (University of Leicester, UK) elaborated on recent investigations into the size and composition of the Apaf-1 apoptosome, which he refers to as the aposome. ${ }^{10}$ THP.1 cell lysates were activated with dATP to induce apoptosis, and were then fractionated by size exclusion chromatography and analyzed for DEVDase activity. Such activity eluted as $700 \mathrm{kDa}$ and 200$300 \mathrm{kDa}$ complexes, as well as a minute amount of $60 \mathrm{kDa}$ free caspase-3. Western blot analysis indicated that the $700 \mathrm{kDa}$ aposome consisted of Apaf-1, and the processed subunits of activated caspase- 9 , caspase- 3 and caspase-7. Activated caspase- 3 and -7 were also present in the 200-300 kDa microaposome. There was also a small amount of free processed caspase-3, -7 and -9 . In non-apoptotic control cells, in contrast, Apaf-1 purified almost exclusively with the microaposome, as did inactive caspase-7, while caspase- 9 and -3 were present as free, inactive proenzymes. When the caspase inhibitor zVADfmk was added to apoptotic lysates, Apaf-1, but not the caspases, was recruited to the aposome. Thus, upon induction of apoptosis, Apaf-1 forms large, multimeric complexes that include caspase-9, caspase-3, and caspase-7. This provides biochemical evidence, using endogenous cell components, for the induced-proximity model of activation of the initiator caspase- 9 by Apaf- $1 .{ }^{9,11}$ It further implies that the effector caspases, caspase-3 and -7, are also intrinsic components of the apoptosome. These findings raise interesting questions for future investigation, such as: are there additional proteins present in the aposome? What is the function of the microaposome? Does it represent the next step in the caspase cascade, activation of downstream effector caspases, or perhaps it is involved in targeting the catalytic activity to specific cellular substrates?

Cohen mentioned that Hsp90 was also present in the microaposome, and may be involved in targeting activated caspases to their substrates. This is an interesting 
observation, in light of recent articles proposing a role for additional heat shock proteins, Hsp60 and Hsp10, in facilitating the processing of pro-caspase-3. ${ }^{12,13}$ Although some heat shock proteins may facilitate caspase activation, others appear to block caspase activity. Douglas Green showed that Hsp70 can block activation of caspase-9 and caspase-3 after addition of cytochrome $c$ and dATP to cell extracts. This activity required an intact peptide binding domain. Hsp70 appears to block formation of the apoptosome; in the presence of Hsp70, endogenous caspase- 9 and caspase- 3 remain as free, inactive zymogens, instead of co-fractionating as processed subunits with the larger molecular weight complex containing Apaf-1. Hsp70 can bind Apaf-1, in an in vitro sepharose pull down assay, and binding is improved in the presence of cytochrome $c$ and dATP.

Hermann Steller (Massachusetts Institute of Technology, USA) described the Drosophila homolog of Apaf-1, known as HAC-1. ${ }^{14}$ It contains the Ced-4 homology domain, as well as the WD-40 repeats, but lacks a CARD domain. It can activate the Drosophila caspases drICE and DCP-1 in a dATP-dependent manner. Hac-1 is transcriptionally upregulated in response to radiation, and loss of function mutations in hac-1 lead to diminished developmental cell death in some cell types. Thus, this part of the mammalian death machinery is conserved in Drosophila, and represents a second pathway, independent of reaper, hid and grim (see below) that leads to caspase activation. Although much progress has been made in understanding the mitochondrial-dependent apoptotic pathway through biochemical assays such as the ones described above, the discovery of the Drosophila counterparts will enable a molecular genetic approach to further define the pathway.

\section{Death receptor dependent caspase activation}

The death receptor pathway for activation of caspases was one of the first apoptotic pathways elucidated. ${ }^{15}$ It involves formation of an apoptosome, also referred to as the DISC, at the plasma membrane. Binding of death ligands such as FasL or $\mathrm{TNF} \alpha$ to their respective receptors induces receptor trimerization and activation. The activated receptor then binds adaptor molecules such as FADD and TRADD through common death domain (DD) motifs. These adaptors, in turn, recruit the initiator caspase-8, by interactions through the death effector domains (DED). Oligomerized caspase-8 undergoes auto-catalysis and initiates the caspase proteolytic cascade. The basic framework of the signaling mechanism has been known for several years now, thanks in part, to landmark work done by participants of the meeting, including David Wallach (Weizmann Institute of Science, Israel) and Peter Krammer (German Cancer Research Center, Heidelberg, Germany). Focus has been shifted now to fine-tuning the details of the model.

Wallach presented an informative overview of the various pathways activated by death receptors, subjecting each to a fidelity test for credibility. His talk was provocative and raises an important point: the apoptosis field, after more than a decade of research, has accumulated a large quantity of data that must be sorted through to identify what is known and proven, what is physiologically relevant to an in vivo setting, what represents artifacts of over-expression systems, and what remains as speculative models. For example, the direct pathway from Fas to FADD to caspase8 fulfills the stringent criteria for authenticity, as the recent knock-out mice prove. ${ }^{16,17}$ In contrast, less evidence exists in support of a role for caspase-10 in death receptormediated apoptosis, although human mutations in caspase10 have been associated with reduced death receptorinduced apoptosis and auto-immune disease. ${ }^{18}$ Similarly, the physiological relevance of the TNFR-TRADD-RIPRAIDD-caspase-2 pathway has not been established, as both RIP $^{-1-}$ and caspase- $2^{-1-}$ mice are sensitive to TNFinduced apoptosis. ${ }^{19,20}$

Some interesting observations concerning the death receptor pathways in specific cell systems were discussed by Peter Krammer. He reviewed data about the difference between T cells on day 1 and day 6 following activation, only the latter of which are responsive to Fas-induced apoptosis. Previously, it had been emphasized that the difference between these two cell types was the level of FLIP expression. ${ }^{21}$ FLIP is a caspase-8 like protein that lacks key residues important for catalysis. While some studies have determined that over-expression of FLIP induces apoptosis, others have proposed that FLIP is a negative regulator of the Fas pathway. ${ }^{22}$ In fact, the reduction in FLIP expression from day 1 to day 6 following activation of $\mathrm{T}$ cells served as physiologically relevant evidence in favor of the latter hypothesis. ${ }^{21}$ Krammer, however, revisited this point and stated that he observed little change in FLIP $P_{L}$ expression, although FLIP $_{S}$ did decrease in the Fas-sensitive day 6 cells.

Krammer has searched for other differences between day 1 and 6 activated $T$ cells. In general, cells can be divided into two categories according to their phenotypic response to death receptor activation. While certain cells (Type I) rely predominantly on the direct Fas-caspase-8 apoptosome to activate effector caspases, other cells (Type II), in which only a limited amount of activated caspase-8 is formed, rely on the cleavage of Bid and the activation of caspase -9 to amplify the apoptotic signal. ${ }^{23}$ Day 6 activated $T$ cells form a proper DISC containing caspase-8 and FADD (and also FLIP), thus behaving like Type I cells in which the death receptor pathway is the major mechanism of activating the caspase cascade. Day $1 \mathrm{~T}$ cells, on the other hand, do not form a proper DISC and resemble Type II cells. Significantly, in contrast to day 6 cells, day 1 cells express high levels of $\mathrm{Bcl}-\mathrm{x}_{\mathrm{L}}$. Thus formation of both types of apoptosome is inhibited in day 1 resistant $T$ cells, due to the combination of limited DISC assembly and high $\mathrm{Bcl}-\mathrm{x}_{\mathrm{L}}$ expression.

\section{A novel apoptosome}

Considering the large number of molecules recently identified that contain CARD or death domains, ${ }^{24,25}$ it is not too surprising to suggest that novel apoptosomes may exist in the cell. In fact, Seamus Martin described an alternate pathway to activate caspase-9. The CARD domain of caspase- 9 was used in a yeast- 2 hybrid screen to identify 
two interacting proteins, CARP-1/DRADD and CARP-2. DRADD contains a central CARD domain and a C-terminal death domain, suggesting it serves as an adaptor protein. In support of its specific role in caspase-9 mediated death, a dominant negative DRADD (lacking the CARD domain) did not block apoptosis induced by over-expression of death receptors. Thus DRADD may define a novel pathway by which caspase- 9 and the caspase cascade can be activated.

\section{The execution stage: caspases at work}

\section{Caspase substrates}

Following activation, the effector caspases cleave specific cellular substrates, facilitating breakdown of the apoptotic cell. Although many putative caspase substrates have been identified, the significance of only a few of these substrates has been demonstrated. ${ }^{1,22}$ As David Wallach stressed, proposed caspase substrates must also be subjected to a fidelity test to determine which of the many proteins shown to be caspase substrates are actually involved in, and necessary for, the execution of the apoptotic phenotype.

One important caspase substrate is ICAD/DFF45, the inhibitor of CAD/DFF40, a DNase responsible for the internucleosomal fragmentation of chromosomal DNA. ${ }^{26-29}$ Shigekazu Nagata (Osaka University Medical School, Japan) discussed the regulation of CAD by ICAD, which involves two mechanisms. ICAD binds to and inhibits CAD activity, until ICAD is cleaved by caspase- 3 or caspase- 7 , whereupon the active CAD nuclease is released from the complex. Furthermore, ICAD , but not a second isoform, ICAD , exhibits specific chaperone-like activity towards CAD, enabling proper folding of the protein. ${ }^{30}$ Nagata also described the isolation of a Drosophila ortholog of CAD, which he refers to as dCAD. Unlike the mammalian counterparts, dCAD contains no nuclear localization signal and requires cleavage by caspases in order to be activated.

Nagata generated transgenic mice expressing a dominant negative form of ICAD that lacks the caspase cleavage sites that are required for activation of CAD. ${ }^{31}$ In vivo analysis of apoptosis in this model revealed an interesting CAD-independent mechanism of DNA degradation. TUNEL positivity was still observed in cells undergoing spontaneous apoptosis. In fact, upon whole animal $\gamma$ irradiation, TUNEL positive cells were observed exclusively within phagocytic macrophages. In these cells, DNA fragmentation was mediated by a macrophage acidsensitive lysosomal DNase. Thus, a second, cell nonautonomous mechanism for degradation of chromosomal content exists in mammals, similar to that originally attributed to the $C$. elegans nuc1 endonuclease, which functions in the engulfing cell. ${ }^{32}$

A second caspase substrate discussed at the meeting was $\mathrm{p} 27^{\mathrm{kip} 1}$. Eric Solary (INSERM, Dijon, France) presented evidence for cleavage of $p 27^{\text {kip } 1}$ by caspases during apoptosis. ${ }^{33}$ Caspases-3 and -8 are capable of cleaving the protein in vitro. Over-expression of p27 $7^{\mathrm{kip} 1}$ in U937 cells causes a delay in etoposide-induced activation of caspases. Similarly, expression of the caspasegenerated cleavage fragments of p2 $7^{\mathrm{kip} 1}$ inhibits etopo- side-induced death, while a cleavage-resistant mutant has no such inhibitory effect. This suggests that $\mathrm{p} 27^{\mathrm{kip} 1}$ may be involved in a negative feedback loop, whereby caspasemediated cleavage of the protein produces fragments that inhibit further caspase activation. In this manner, p27 $7^{\text {kip } 1}$ may contribute to tumor resistance to chemotherapeutic drugs.

\section{Localization of caspases}

Recent work has highlighted one aspect of the regulation of the execution stage of cell death, that is, subcellular localization of activated caspases. ${ }^{34-36}$ Gerald Cohen used GFP-fused caspases to study the subcellular localization of caspases following TRAIL-induced apoptosis in MCF-7 cells. In control cells, caspase-3, -7 and -9 were cytoplasmic. Upon induction of apoptosis, however, caspase-9 assumed a punctate distribution, while caspase- 3 and -7 were localized to cytoplasmic inclusions. Further analysis using EM and immunocytochemistry indicated that these inclusions did not consist of ER, mitochondria or the actin cytoskeleton. However, the inclusions did contain cytokeratins-8, -18 , and -19 , as well as a caspase cleavage product of cytokeratin-18. Thus, the inclusions observed may represent sites where caspase-3 is targeted to at least one of its substrates, cytokeratin.

Sharad Kumar (University of Adelaide, Australia) discussed the localization and regulation of caspase-2. Full length caspase-2 possesses both a cytoplasmic and nuclear distribution. The latter is mediated by the prodomain, which consists of a CARD domain and a linker region that connects it to the catalytic domain. The linker appears to act as a negative regulator; if one deletes just the CARD domain, caspase-2 is inactive, however a further deletion of the linker region restores its catalytic and killing activities. The pro-domain also mediates homo-dimerization, and can confer the ability to dimerize and auto-activate when fused to caspase- 3 . $^{37,38}$

\section{SIGNALING TO THE DEATH MACHINERY}

It is clear that the death machinery of every cell must be tightly controlled, such that it can be activated precisely, and only when required. Signaling for suicide generally involves both inactivation of inhibitors ('taking off the brakes') as well as induction of effectors ('foot on the accelerator'). In this way, there are many possible levels of control and multiple, sometimes conflicting, signals can be integrated.

\section{Regulation of the Fas death receptor pathway}

Death receptor signaling, as described above, is the most direct pathway from the plasma membrane to caspase activation and the time interval between ligand-receptor binding and death can be as little as a few hours. There is much evidence to indicate that the death receptor pathway is part of, though not necessarily required for, pro-apoptotic signaling cascades initiated by diverse stimuli. For example, Peter Krammer summarized evidence suggesting that Fas 
signaling plays a role in the apoptotic response of tumor cells to chemotherapeutic drugs. Expression of both FasL and Fas are increased at the level of transcription. In the case of FasL, this increase is dependent on activity of the transcription factor c-jun, which is activated by the JNK signaling pathway, while for Fas, it is mediated by the transcriptional transactivating function of $553 .^{39}$ Similarly, Cristopher $E$ Henderson (Developmental Biology Institute of Marseille, France) discussed the relevance of the Fas signaling pathway to trophic factor-dependent death of developing rat embryonic motoneurons. He found that soluble Fas and a peptide inhibitor of caspase-8 blocked trophic factor deprivationinduced death. Moreover, in the presence of trophic factor, $50 \%$ of motoneurons died in response to anti-Fas antibody. The remaining cells were resistant to killing by Fas, most likely due to the upregulation of FLIP. ${ }^{40}$ This data supports a significant role for Fas signaling in apoptosis induced by survival factor withdrawal. In contrast to Fas, which is expressed both in the presence and absence of growth factors, FasL expression is increased in the absence of trophic factors. This is facilitated by two signaling pathways that regulate FasL transcription. One involves JNK-mediated activation of c-jun, as described above. The second pathway involves FKHRL1, a member of the forkhead family of transcription factors, which binds and activates the FasL promoter. In the presence of survival factors, Akt/protein kinase B-mediated FKHRL1 phosphorylation leads to inactivation of FKHRL1 and causes its sequestration to the cytoplasm. Upon withdrawal of trophic factors, however, PI3K activation of Akt is inhibited, thus enabling the transcription factor FKHRL1 to transactivate FasL. ${ }^{41}$

\section{Signaling by the p53 family}

There can be no meeting on apoptosis without some mention of the tumor suppressor p53, which is mutated in over half of human cancers. The $\mathrm{p} 53$ protein is recognized as an essential component of signaling pathways that respond to various forms of cellular stress. It has been known for a few years that, in addition to cell cycle arrest, another important biological outcome of p53 function is apoptosis. ${ }^{42}$ Nevertheless, it is still unclear when, and by what precise mechanism(s), p53 signals to activate the death machinery. p53 is reported to regulate the death machinery in several ways, both at the transcriptional and post-transcriptional level, but the importance of each control point, and of cell type/environment, is the subject of active research. Douglas Green described a system whereby p53-dependent apoptosis is triggered by adenoviral expression of p53 in Saos-2 cells, which are p53null. An early event in this apoptotic process was zVAD-fmkinsensitive, mitochondrial cytochrome $c$ release. Addition of cytosolic extracts prepared from these p53-transduced, apoptotic cells to isolated mitochondria induced release of cytochrome $c$ in a Bax-dependent manner. Extracts from cells infected with control virus had no such effect. This data suggests that there is a p53-dependent activation of Bax. This activation of Bax did not require p53 transcriptional activity or nuclear localization. Recombinant Bax alone was more efficient in inducing cytochrome $c$ release from isolated mitochondria than recombinant Bax plus normal, untreated cytosol. Furthermore, the addition of cytosol from the apoptotic cells to recombinant Bax restored efficient cytochrome $c$ release. On the basis of these results, Green hypothesizes that p53 relieves an antagonistic activity in the cytoplasm that inhibits the ability of Bax to induce cytochrome $c$ release, and in this way p53 turns on the death machinery. ${ }^{43}$

Another area of active p53 research concerns the components upstream to p53 that transmit the stress signal to p53. Moshe Oren (Weizmann Institute of Science, Israel) presented evidence concerning two proteins that he has identified that signal to $\mathrm{p} 53$, namely $\beta$-catenin and Werner protein (WRN). He described the ability of $\beta$-catenin to upregulate p53 protein levels and transcriptional activity. ${ }^{44}$ In light of this data, it is conceivable that upregulation of $\beta$-catenin might pose a selective pressure for cells to abrogate p53 function. This hypothesis could explain the observation that mutational inactivation of p53 at late stages of colorectal cancer generally follows $\beta$-catenin deregulation, which occurs at an earlier stage. Experiments to address whether $\beta$-catenin modulates p53 apoptotic activity are in progress. WRN is the product of a gene which, when mutated, results in the Werner premature aging syndrome. WRN can physically associate with p53 and, similar to $\beta$-catenin, has the capacity to increase p53 protein levels and transcriptional activity. ${ }^{45}$ New data indicates that WRN is important for the apoptotic function of $\mathrm{p} 53$.

There have been exciting advances in delineating the moleclar mechanism by which signals induce p53 activity. The oncoprotein MDM2 is encoded by a target gene of p53. MDM2 negatively regulates p53 function by binding directly to p53, thereby inhibiting the latter's transcriptional activity and promoting its proteasomal degradation (reviewed in Juven-Gershon and Oren). ${ }^{46}$ Work in various systems suggests that this MDM2-p53 feedback loop serves as an integration point of stress signals, which is modulated in several ways. Moshe Oren discussed experiments which provide evidence for three methods of regulating the MDM2-p53 interaction. Mutational analysis indicated that phosphorylation of p53 on the Ser-20 residue within the MDM2 binding site inhibits association between the two proteins and thus results in p53 accumulation. ${ }^{47}$ Importantly, phosphorylation at this site is increased after DNA damage. ${ }^{48}$ This data exemplifies method 1 , where modification of p53 blocks binding to MDM2. Work done in collaboration with Yossi Shiloh (Weizmann Institute of Science, Israel) demonstrates method 2, which involves modification of MDM2, such that its ability to induce p53 degradation is impaired. ${ }^{49}$ MDM2 was shown to be phosphorylated in response to some types of DNA damage. This ATM (ataxia telangiectasia mutated protein)-dependent phosphorylation was found not to affect p53-MDM2 binding, but rather to reduce the ability of MDM2 to promote p53 degradation. Finally, method 3 involves modulation of MDM2 levels in response to stimuli, resulting in a shift in the outcome of the p53-MDM2 loop. Moshe Oren described collaborative experiments carried out with Abraham Amsterdam (Weizmann Institute of Science, Israel), that correlate exposure to basic FGF 
survival factor with increased MDM2 protein level and concomitant downregulation of p53 activity. ${ }^{50,51}$

Until recently, p53 was thought to be the only gene of its kind in vertebrate genomes. However, this widely held conviction has been disproved. Two genes, referred to as p63 and p73, have been found to encode proteins that share significant amino acid identity with p53 in the transactivation domain, the DNA binding domain, and the oligomerization domain (for review see Levrero et al). ${ }^{52}$ Despite this homologous relationship, mouse knock out analysis of the function of these three family members indicates that each plays quite different biological roles. ${ }^{53-55}$ Nevertheless, there is evidence to indicate that these proteins do share some biological activities. For example, like p53, p73 can induce apoptosis. Gerry Melino (IDI-IRCCS Biochemistry Lab., Italy) reviewed his recent report that the tyrosine kinase c-Abl regulates p73 in the apoptotic response to cisplatininduced DNA damage, by increasing the half-life of the p73 protein. ${ }^{56}$ This stabilization requires c-Abl kinase activity, although increased Tyr phosphorylation was not observed. Work with cells defective for mismatch repair indicated an additional requirement for the mismatch repair gene MLH1 in apoptotic signaling to $\mathrm{p} 73$. Two other teams have reported results in other systems that support the proposed signaling pathway from c-Abl to p73, and have shown that c-Abl and p73 interact directly. ${ }^{57,58}$ Some notable differences between the data from these groups suggests that there may be signal and/or cell specific variations in the molecular mechanism of c-Abl signaling to p73.

\section{Signaling pathways in Drosophila}

Herman Steller described work in Drosophila that has revealed another signaling pathway that controls caspase activation, probably independently of the others. Genetic studies have shown that three adjacent genes, reaper, grim and hid, have central roles in the control of apoptosis induction (for review see Bergmann et al). ${ }^{59}$ Flies homozygous for a deletion that removes all three genes display virtually no apoptosis during development and die as embryos. These three genes are transcriptionally regulated in response to diverse death-inducing signals, as manifested by their complex promoters that contain many transcription factor binding sites. Additionally, an elegant genetic screen for genes that modify the phenotype of hid expression in the eye indicated that HID is posttranscriptionally regulated by the Ras-MAP kinase pathway in response to survival signals generated from the EGF receptor. ${ }^{60}$ Steller further described examination of gain of function mutations in one of the Drosophila IAPs (inhibitors of apoptosis), diap1, which strongly suppressed reaper-, hid- and grim-induced apoptosis. IAPs have been shown to directly bind to and inhibit caspases. ${ }^{61}$ REAPER, HID and GRIM can interact physically with the IAPS, which antagonizes the formers' pro-apoptotic activities. ${ }^{62}$ The gain of function DIAP1 mutants identified in the genetic screen displayed reduced binding to REAPER, HID and GRIM. Furthermore, simultaneous mutation of reaper, grim and hid and diap1 resulted in excessive apoptosis, similar to loss of diap1 alone. These and other observations support a 'double-negative' model, in which REAPER, HID and GRIM inhibit the activity of IAPs, which in turn block caspase activity. ${ }^{63}$ Orthologues of reaper, grim and hid have not yet been identified in higher organisms, but their over-expression promotes apoptosis in mammalian cells, suggesting there may be conservation of this signaling pathway. $^{64}$

\section{The apoptotic network}

As our understanding of the central players of the apoptotic machinery advances, it becomes clear that only a fraction of the signaling pathways that regulate the initiation and execution of apoptosis have been uncovered. These will involve an intricate network of cross-talk between the known pathways described above, as well as additional, novel pathways still to be identified and characterized. Several groups have attempted to expand this network by undertaking unbiased genetic screens in mammalian cells to identify novel regulators and components of the death machinery.

Adi Kimchi (Weizmann Institute of Science, Israel) demonstrated the power of one such screen, termed the technical knockout (TKO). In this screen, HeLa cells expressing an episomal, antisense cDNA library were selected for survival in the presence of interferon- $\gamma$. Five genes whose expression was necessary for interferon- $\gamma$ induced cell death were identified in this manner, including DAP-kinase (DAP-k) and DAP5. ${ }^{65}$ DAP-k is a $\mathrm{Ca}^{2+} /$ calmodulin-regulated Ser/Thr kinase, containing multiple functional domains, including a cytoskeletal binding region, a death domain and a C-terminal Ser rich tail. Overexpression of DAP-k induces apoptosis, and DAP-k activity is necessary for apoptosis induced by a variety of stimuli. The death domain is necessary for its death-promoting activity, while the Ser-rich tail acts as a negative regulator. ${ }^{66}$ Consistent with its role as a regulator of apoptosis, DAP-k exhibits tumor suppressor properties. Loss of DAP-k expression has been documented in highly metastatic lung carcinomas and in various neoplastic human cell lines, and tumor growth and metastasis are suppressed by reintroduction of DAP-k to physiological levels. ${ }^{67}$ There is evidence of loss of heterozygosity and transcriptional silencing through methylation in various colon and breast tumor samples analyzed. Additionally, DAP-k suppresses transformation by myc/ras and E1A/ras in cultured embryonic fibroblasts in a p53- and p19 arf dependent manner. Thus, DAP-k functions as a proapoptotic protein that is involved in regulating apoptotic events common to multiple death stimuli, and as such, plays a role both in early transformation checkpoints and later metastatic events.

The identification of DAP5, a homolog of the translation initiation factor elF4G, highlights the importance of the regulation of translation to apoptosis. Unlike elF4G, DAP5 lacks the region of the protein necessary for interaction with the cap-recognition factor elF4E. Thus, it is predicted to mediate cap-independent initiation only. In fact, cell free translation assays using rabbit reticulocyte lysates indicate that DAP5 mediates cap-independent translation from an internal ribosome entry site (IRES) located in the DAP5 5' 
UTR. Following various apoptotic stimuli, the $97 \mathrm{kDa}$ DAP5 protein is cleaved by a caspase to form an $86 \mathrm{kDa}$ product. This 86 kDa form exhibits greater IRES-dependent translational activity compared to the full-length protein in cell free translation assays. Significantly, the translation of DAP5 protein, as well as a reporter gene under the control of the DAP5 IRES, is selectively maintained during apoptosis, even as the overall rate of translation falls. This suggests that DAP5 utilizes IRES-dependent translation to autoregulate its expression. ${ }^{68}$ These data reveal a novel layer of regulation during apoptosis, whereby genes whose functions are necessary for the execution of, or conversely, the protection from, apoptosis are specifically translated through cap-independent means. Interestingly, three known apoptosis-related genes, c-myc, xiap and apaf-1, have been recently shown to contain IRES sequences that may potentially maintain their translation during apoptosis. ${ }^{69-71}$ A search for DAP5 target genes will shed much light on the mechanism by which translational control regulates apoptosis.

Louis Deiss (University of Illinois, Chicago, USA) described the Achilles Heel Method to select for genes whose deletion sensitizes cells to sub-optimal death signals, specifically, a sub-lethal dose of anti-Fas antibody. Using this method, he identified several known genes, as well as several novel genes. He described two at the meeting - FGF and NF-E2 related factor (Nrf2). Nrf2 is a transcription factor involved in activating phase II detoxifying enzymes, such as glutathione-S transferase, in response to carcinogens and xenobiotics. In fact, induction of the phase II response by addition of glutathione can protect cells from Fas-induced apoptosis. The known prosurvival activity of FGF lends support to its discovery in such a screen, and provides proof-of-concept for the methodology described. However, the identification of a factor involved in detoxification raises a warning flag concerning interpretation of such screens. Distinctions must somehow be made between gene products and pathways that are involved genuinely in the signaling of apoptosis, and those that are unrelated to apoptosis, although important for cell homeostasis. This will be a particularly important point to keep in mind as the apoptotic network expands to include new pathways and regulators.

\section{NON-CONVENTIONAL (CASPASE- INDEPENDENT) DEATH PATHWAYS}

Cell death is often categorized, both functionally and morphologically, into two types: (1) apoptosis - an active, genetically programmed, caspase-dependent cell death accompanied by cell shrinkage, nuclear condensation and phagocytic uptake, versus (2) necrosis - a passive, unprogrammed cell death associated with cytoplasmic swelling, membrane rupturing and organelle dissolution. This simple, popular categorization needs to be re-evaluated and new types defined in order to avoid semantic confusion. There is accumulating evidence to indicate the existence of a genetically programmed cell death pathway(s) that does not involve caspase activation, in which cells exhibit a mixture of 'necrotic' and 'apoptotic' phenotypic features (for reviews and discussion see s2-76 $^{7}$ Several presentations related to such alternative caspase-independent cell death.

Loss of interdigital cells in the mouse embryo, a paradigm of cell death during development, is known to include an apoptotic and caspase-dependent mechanism. Pierre Golstein (Centre d'Immunologie de Marseille-Luminy, France) reported that when caspase activity was compromised, as a result of either caspase inhibition or genetic mutations, interdigital cell death still occurred in mouse limb buds. This cell death was reminiscent of necrosis in that it involved mitochondrial and membrane lesions without detectable DNA cleavage, although it lacked the typical necrosis-associated inflammatory response. Importantly, cells with similar morphology were observed by electron microscopic analysis in wild-type, untreated limb buds. These data suggest that alongside the more classical caspase-dependent cascade leading to apoptosis, there is a programmed, caspase-independent cascade of events that might contribute to elimination of interdigital cells. ${ }^{77}$

Although such morphological analysis of developmental cell death suggests that cell death with necrotic morphology may be active and programmed, an intrinsic 'necrotic' death program can only be proven by identification of genes that regulate and mediate it. One such gene may be the ras oncogene, which is rarely mutated in certain tumor types. Yoshiyuki Kuchino (National Research Cancer Institute, Japan) found that introduction of oncogenically mutated ras into human glioma or gastric tumor cells induced cellular degeneration and nuclear pyknosis accompanied by cytoplasmic lysosomal-derived vacuoles, suggestive of autophagy. ${ }^{78}$ This death occurred in the absence of caspase activation and was not inhibited by over-expression of the anti-apoptotic Bcl-2 protein. Wild-type ras triggered death, albeit less efficiently. These results support a role for Ras in transducing necrotic-like death signals, and explain why there is little selective advantage for tumors of these types to acquire mutations in ras. Further analysis indicated that PI3K, a physiological effector of Ras, plays an essential role in this Ras-mediated cell death. Shigekazu Nagata (Osaka University Medical School, Japan) mentioned work carried out in his laboratory that also points to a programmed necroticlike cell death. Forced dimerization of Fas or FADD in a Jurkat cell line expressing high levels of $\mathrm{Bcl}-2$ were shown to cause caspase-independent cell death, accompanied by cell swelling and lacking DNA fragmentation. ${ }^{79}$

This relatively new area of caspase-independent death pathways is likely to be a primary focus of interest in the cell death field that we are sure to hear more about at future meetings. It will be important to reveal the molecular regulators of this non-classical pathway. A key aim of cancer therapy is to trigger cancer cell suicide. Research into delineation of a 'non-apoptotic' genetic program of cell death, in addition to furthering our understanding of the classical apoptotic one, should enable new combinatorial therapeutic approaches that manipulate both pathways. In this way, whichever pathway remains intact in the tumor cell, there is increased probability of successfully triggering cell suicide. 


\section{FINAL COMMENTS}

The thought provocative meeting at Ein Gedi touched on some of the crucial, exciting areas of current interest in apoptosis. Clearly the central apoptotic machinery, of which we now have generated a fairly detailed understanding, is only one spoke of the wheel that regulates cell death. We look forward to future meetings to hear more about the additional spokes and the means by which they interconnect.

\section{References}

1. Nicholson D (1999) Caspase structure, proteolytic substrates, and function during apoptotic . Cell Death Differ. 6: 1028-1042

2. Luo X, Budihardjo I, Zou HC, Slaughter C and Wang X (1998) Bid, a Bcl2 interacting protein, mediates cytochrome $c$ release from the mitochondria in response to cell surface death receptors. Cell 94: 481-490

3. Li H, Zhu H, Xu-C-J and Yuan J (1998) Cleavage of BID by caspase 8 mediates the mitochondrial damage in the Fas pathway of apoptosis. Cell 94: 491-501

4. Martinou I, Desagher S, Eskes R, Antonsson B, Andre E, Fakan Sand Martinou JC (1999) The release of cytochrome $c$ from mitochondria during apoptosis of NGF-deprived sympathetic neurons is a reversible event. J. Cell Biol. 144: 883889

5. Goldstein J, Waterhouse N, Juin P, Evan G and Green D (2000) The coordinate release of cytochrome $c$ during apoptosis is rapid, complete and kinetically invariant. Nature Cell Biol. 2: 156-162

6. Desagher S, Osen-Sand A, Nichols A, Eskes R, Montessuit S, Lauper S, Maundrell K, Antonsson B and Martinou J-C (1999) Bid-induced conformational change of $\mathrm{Bax}$ is responsible for mitochondrial cytochrome $\mathrm{c}$ release during apoptosis. J. Cell Biol. 144: 891-901

7. Gross A, Jockel J, Wei MC and Korsmeyer SJ (1998) Enforced dimerization of $B A X$ results in its translocation, mitochondrial dysfunction and apoptosis. EMBO J. 17: $3878-3885$

8. Adrain C, Slee E, Harte M and Martin S (1999) Regulation of apoptotic protease activating factor-1 oligomerization and apoptosis by the WD-40 repeat region. J. Biol. Chem. 274: 20855-20860

9. Srinivasula SM, Ahmad M, Fernandes-Alnemri T and Alnemri ES (1998) Autoactivation of procaspase- 9 by Apaf-1-mediated oligomerization. Mol. Cell. 1: $949-957$

10. Cain K, Brown DG, Langlais C and Cohen GM (1999) Caspase activation involves the formation of the aposome, a large $(\sim 700 \mathrm{kDa})$ caspase-activating complex. J. Biol. Chem. 274: 22686-22692

11. Li P, Nijhawan D, Budihardjo I, Srinivasula SM, Ahmad M, Alnemri ES and Wang X (1997) Cytochrome $c$ and dATP-dependent formation of Apaf-1/caspase-9 complex initiates an apoptotic protease cascade. Cell 91: 479-489

12. Samali A, Cai J, ZhivotovskyB, Jones D and Orrenius S (1999) Presence of a preapoptotic complex of pro-caspase-3, Hsp60 and Hsp10 in the mitochondrial fraction of jurkat cells. EMBO J. 18: 2040-2048

13. Xanthoudakis S, Roy S, Rasper D, Hennessey T, Aubin Y, Cassady R, Tawa $P$ Ruel R, Rosen A and Nicholson D (1999) Hsp60 accelerates the maturation of pro-caspase- 3 by upstream activator proteases during apoptosis. EMBO J. 18 2049-2056

14. Zhou L, Song Z, Tittel J and Steller H (1999) HAC-1, a Drosophila homolog of Apaf- 1 and ced-4, functions in developmental and radiation-induced apoptosis Mol. Cell. 4: 745-755

15. Ashkenazi A and Dixit VM (1998) Death receptors: signaling and modulation Science 281: 1305-1308

16. Yeh W-C, de la Pompa J, McCurrach M, Shu H, Elia A, Shahinian A, Ng M, Wakeham A, Khoo W, Mitchell K, El-Deiry W, Lowe S, Goeddel D and Mak T (1998) FADD: essential for embryo development and signaling from some, but not all, inducers of apoptosis. Science 279: 1954-1958

17. Varfolomeev E, Schuchman M, Luria V, Chiannilkulchai N, Beckman J, Mett I Rebrikov D, Brodianski V, Kemper O, Kollet O, Lapidot L, Soffer D, Sobe T, Avraham K, Goncharov T, Holtmann H, Lonai P and Wallach D (1998) Targeted disruption of the mouse caspase 8 gene ablates cell death induction by the TNF receptors, Fas/Apo1, and DR3 and is lethal prenatally. Immunity 9: 267-276
18. Wang J, Zheng L, Lobito A, Chan F, DaleJ, Sneller M, YaoX, PuckJ, Straus S and Lenardo M (1999) Inherited human Caspase 10 mutations underlie defective lymphocyte and dendritic cell apoptosis in autoimmune lymphoproliferative syndrome type II. Cell 98: 47-58

19. Bergeron L, Perez GI, Macdonald G, Shi L, Sun Y, Jurisicova A, Varmuza S, Latham KE, Flaws JA, Salter JC, Hara H, Moskowitz MA, Lie E, Greenberg A, Tilly $\mathrm{JL}$ and Yuan J (1998) Defects in regulation of apoptosis in caspase-2-deficient mice. Genes Dev. 12: $1304-1314$

20. Kelliher M, Grimm S, Ishida Y, Kuo F, Stanger B and PL (1998) The death domain kinase RIP mediates the TNF-induced NF-kappaB signal. Immunity 8: 297-303

21. Irmler M, Thome M, Hahne M, Schneider P, Hofmann K, Steiner V, Bodmer JL, Schroter M, Burns K, Mattmann C, Rimoldi D, French LE and Tschopp J (1997) Inhibition of death receptor signals by cellular FLIP. Nature 388: 190-195

22. Cryns V and Yuan J (1998) Proteases to die for. Genes Dev. 12: 1551 - 1570

23. Scaffidi C, Fulda S, Srinivasan A, Friesen C, Li F, Tomaselli KJ, Debatin KM, Krammer PH and Peter ME (1998) Two CD95 (APO-1/Fas) signaling pathways. EMBO J. 17: 1675-1687

24. Kumar S (1999) Mechanisms mediating caspase activation in cell death. Cell Death Differ. 6: 1060-1066

25. Cecconi F (1999) Apaf1 and the apoptotic machinery. Cell Death Differ. 6: 1087 1098

26. Liu X, Li P, Widlak P, Zou H, Luo X, Garrard WT and Wang X (1998) The 40-kDa subunit of DNA fragmentation factor induces DNA fragmentation and chromatin condensation during apoptosis. Proc. Natl. Acad. Sci. USA 95: 8461-8466

27. Liu X, Zou H, Slaughter $C$ and Wang X (1997) DFF, a heterodimeric protein that functions downstream of caspase- 3 to trigger DNA fragmentation during apoptosis. Cell 89: 175-184

28. Sakahira H, Enari M and Nagata S (1998) Cleavage of CAD inhibitor in CAD activation and DNA degradation during apoptosis. Nature 391: 96-99

29. Enari M, Sakahira H, Yokoyama H, Okawa K, Iwamatsu A and Nagata S (1998) A caspase-activated DNase that degrades DNA during apoptosis, and its inhibitor ICAD. Nature 391: 43-50

30. Sakahira H, Iwamatsu A and Nagata S (2000) Specific chaperone-like activity of inhibitor of caspase-activated DNase for caspase-activated DNase. J. Biol. Chem. 275: $8091-8096$

31. Mcllroy D, Tanaka M, Sakahira H, Fukuyama H, Suzuki M, Yamamura K, Ohsawa Y, Uchiyama Y and Nagata S (2000) An auxiliary mode of apoptotic DNA fragmentation provided by phagocytes. Genes Dev. 14: 549-558

32. Ellis HM and Horvitz HR (1986) Genetic control of programmed cell death in the nematode C. elegans. Cell 44: 817-829

33. Eymin B, Sordet O, Droin N, Munsch B, Haugg M, Van de Craen M, Vandenabecle $P$ and Solary E (1999) Caspase-induced proteolysis of the cyclindependent kinase inhibitor p27Kip1 mediates its anti-apoptotic activity. Oncogene 18: $4839-4847$

34. Chandler JM, Cohen GM and MacFarlane M (1998) Different subcellular distribution of caspase-3 and caspase-7 following Fas-induced apoptosis in mouse liver. J. Biol. Chem. 273: 10815-10818

35. Bialik S, Cryns VL, Drincic A, Miyata S, WollowickAL, Srinivasan A and Kitsis RN (1999) The mitochondrial apoptotic pathway is activated by serum and glucose deprivation in cardiac myocytes. Circ. Res. 85: 403-414

36. Mancini M, Nicholson DW, Roy S, Thornberry NA, Peterson EP, Casciola-Rosen LA and Rosen A (1998) The caspase-3 precursor has a cytosolic and mitochondrial distribution: implications for apoptotic signaling. J. Cell. Biol. 140: 1485-1495

37. Butt A, Harvey N, Parasivam G and Kumar S (1998) Dimerization and autoprocessing of the Nedd2(caspase-2) precursor requires both the prodomain and the carboxyl-terminal regions. J. Biol. Chem. 273: 6763-6768

38. Colussi P, Harvey Nand SK (1998) Prodomain-dependent nuclear localization of the caspase-2(Nedd2) precursor. A novel function for a caspase prodomain. J. Biol. Chem. 273: 24535-24542

39. Muller M, Wilder S, BannaschD, Israeli D, Lehlbach K, Li-Weber M, Friedman SL, Galle PR, Stremmel W, Oren M and Krammer PH (1998) p53 activates the CD95 (APO-1/Fas) gene in response to DNA damage by anticancer drugs. J. Exp. Med. 188: $2033-2045$

40. Raoul C, Henderson C and Pettmann B (1999) Programmed cell death of embryonic motoneurons triggered through the Fas death receptor. J. Cell. Biol. 147: $1049-1062$ 
41. Brunet A, Bonni A, Zigmond M, Lin M, Juo P, Hu L, Anderson M, Arden K, Blenis J and Greenberg M (1999) Akt promotes cell survival by phosphorylating and inhibiting a Forkhead transcription factor. Cell 96: 857-868

42. Gottlieb TM and Oren M (1998) p53 and apoptosis. Semin. Cancer Biol. 8: $359-$ 368

43. Schuler M, Bossy-Wetzel E, Goldstein J, Fitzgerald P and Green D (2000) p53 induces apoptosis by caspase activation through mitochondrial cytochrome $\mathrm{C}$ release. J. Biol. Chem. 275: 7337

44. Damalas A, Ben-Ze'ev A, Simcha I, Shtutman M, Leal JF, Zhurinsky J, Geiger B and Oren M (1999) Excess beta-catenin promotes accumulation of transcriptionally active $p 53$. EMBO J. 18: 3054-3063

45. Blander G, Kipnis J, Leal JF, Yu CE, Schellenberg GD and Oren M (1999) Physical and Functional Interaction between p53 and the Werner's Syndrome Protein. J. Biol. Chem. 274: 29463-29469

46. Juven-Gershon T and Oren M (1999) Mdm2: The ups and downs. Mol. Med. 5 : $71-83$

47. Unger T, Juven-Gershon T, Moallem E, Berger M, Vogt Sionov R, Lozano G, Oren $M$ and Haupt $Y$ (1999) Critical role for Ser20 of human p53 in the negative regulation of $\mathrm{p} 53$ by Mdm2. EMBO J. 18: 1805-1814

48. Shieh SY, Taya Y and Prives C (1999) DNA damage-inducible phosphorylation of $p 53$ at $\mathrm{N}$-terminal sites including a novel site, ser20, requires tetramerization. EMBO J. 18: 1815-1823

49. Khosravi R, Maya R, Gottlieb T, Oren M, Shiloh Y and Shkedy D (1999) Rapid ATM-dependent phosphorylation of MDM2 precedes p53 accumulation in response to DNA damage. Proc. Natl.Acad. Sci. USA 96: 14973-14977

50. Shaulian E, Resnitzky D, Shifman O,Blandino G, Amsterdam A, Yayon A and Oren M (1997) Induction of Mdm2 and enhancement of cell survival by bFGF. Oncogene 15: 2717-2725

51. Hosokawa K, Aharoni D, Dantes A, Shaulian E, Schere-Levy C, Atzmon R, Kotsuji F, Oren M, Vlodavsky I and Amsterdam A (1998) Modulation of Mdm2 expression and $\mathrm{p53}$-induced apoptosis in immortalized human ovarian granulosa cells. Endocrinology 139: 4688-4700

52. Levrero M, Laurenzi VD, Costanzo A, Gong J, Melino G and Wang JY (1999) Structure, function and regulation of p63 and p73. Cell Death Differ. 6: $1146-$ 1153

53. Mills AA, Zheng B, Wang XJ, VogelH, RoopDRand Bradley A (1999)p63is ap53 homologue required for limb and epidermal morphogenesis. Nature 398: $708-$ 713

54. Yang A, Schweitzer R, Sun D, Kaghad M, Walker N, Bronson RT, Tabin C, Sharpe A, Caput D, Crum C and McKeon F (1999) p63 is essential for regenerative proliferation in limb, craniofacial and epithelial development. Nature 398: 714-718

55. Yang A, Walker N, Bronson R, Kaghad M, Oosterwegel M, Bonnin J, Vagner C, Bonnet H, Dikkes P, Sharpe A, McKeon F and Caput D (2000) p73-deficient mice have neurological, pheromonal and inflammatory defects but lack spontaneous tumours. Nature 404: 99-103

56. Gong JG, Costanzo A, Yang HQ, Melino G, Kaelin Jr WG, Levrero M and Wang JY (1999) The tyrosine kinase C-Abl regulates p73 in apoptotic response to cisplatin-induced DNA damage. Nature 399: 806-809

57. Agami R, Blandino G, Oren M and Shaul Y (1999) Interaction of C-Abl and p73alpha and their collaboration to induce apoptosis. Nature 399: 809-813

58. Yuan ZM, Shioya H, Ishiko T, Sun X, Gu J, Huang YY, Lu H, Kharbanda S, Weichselbaum R and Kufe D (1999) p73 is regulated by tyrosine kinase c-Abl in the apoptotic response to DNA damage. Nature 399: 814-817

59. Bergmann A, Agapite J and Steller H (1998) Mechanisms and control of programmed cell death in invertebrates. Oncogene 17: 3215-3223

60. Bergmann A, Agapite J, McCall Kand StellerH (1998) The Drosophila gene hid is a direct molecular target of Ras-dependent survival signaling. Cell 95: 331-341
61. Deveraux QL, Stennicke HR, Salvesen GS and Reed JC (1999) Endogenous inhibitors of caspases. J. Clin. Immunol. 19: 388-398

62. Wang SL, Hawkins CJ, Yoo SJ, Muller HA and Hay BA (1999) The Drosophila caspase inhibitor DIAP1 is essential for cell survival and is negatively regulated by HID. Cell $98: 453-463$

63. Goyal L, McCall K, Agapite J, Hartwieg E and Steller H (2000) Induction of apoptosis by Drosophila reaper, hid and grim through inhibition of IAP function. EMBO J. 19: 589-597

64. Haining WN, Carboy-Newcomb C, Wei CL and Steller H (1999) The proapoptotic function of drosophila hid is conserved in mammalian cells. Proc. Natl. Acad. Sci. USA 96: $4936-4941$

65. Kimchi A (1998) DAP genes: novel apoptotic genes isolated by a functional approach to gene cloning. Biochim. Biophys. Acta 1377: F13-F33

66. Raveh T, Berissi H, Eisenstein M, Spivak T and Kimchi A (2000) A functional genetic screen identifies regions at the $\mathrm{C}$-terminal tail and death-domain of death-associated protein kinase that are critical for its proapoptotic activity. Proc.Natl. Acad. Sci. 97: 1572-1577

67. Inbal B, Cohen O, Polak-Charcon S, Kopolovic J, Vadai E, Eisenbach L and Kimchi A (1997) DAP kinase links the control of apoptosis to metastasis. Nature 390: $180-184$

68. Henis-Korenblit S, Levy-Strumpf N, Goldstaub D and Kimchi A (2000) A novel form of DAP5 protein accumulates in apoptotic cells as a result of caspase cleavage and internal ribosome entry site-mediated translation. Mol. Cell. Biol. 20: $496-506$

69. Coldwell MJ, Mitchell SA, Stoneley M, MacFarlane M and Willis AE (2000) Initiation of Apaf-1 translation by internal ribosome entry. Oncogene 19: 899 905

70. Stoneley M, Chappell SA, Jopling CL, Dickens M, MacFarlane M and Willis AE (2000) C-Myc protein synthesis is initiated from the internal ribosome entry segment during apoptosis. Mol. Cell. Biol. 20: 1162-1169

71. Holcik M, Lefebvre C, Yeh C, Chow T and Korneluk RG (1999) A new internalribosome-entry-site motif potentiates XIAP-mediated cytoprotection. Nat. Cell. Biol. 1: 190-192

72. Borner C and Monney L (1999) Apoptosis without caspases: an inefficient molecular guillotine? Cell Death Differ. 6: 497-507

73. Lorenzo HK, Susin SA, Penninger J and Kroemer G (1999) Apoptosis inducing factor (AIF): a phylogenetically old, caspase-independent effector of cell death. Cell Death Differ. 6: 516-524

74. Samali A, Zhivotovsky B, Jones D, Nagata S and Orrenius S (1999) Apoptosis: cell death defined by caspase activation. Cell Death Differ. 6: 495-496

75. Vaux DL (1999) Caspases and apoptosis - biology and terminology. Cell Death Differ. 6: 493-494

76. Kitanaka C and Kuchino Y (1999) Caspase-independent programmed cell death with necrotic morphology. Cell Death Differ. 6: 508-515

77. Chautan M, Chazal G, Cecconi F, Gruss P and Golstein P (1999) Interdigital cell death can occur through a necrotic and caspase-independent pathway. Curr. Biol. 9: 967-970

78. Chi S, Kitanaka C, Noguchi K, Mochizuki T, Nagashima Y, Shirouzu M, Fujita H, Yoshida M, Chen W, Asai A, Himeno M, Yokoyama S and Kuchino Y (1999) Oncogenic Ras triggers cell suicide through the activation of a caspaseindependent cell death program in human cancer cells. Oncogene 18: 22812290

79. Kawahara A, Ohsawa Y, Matsumura H, Uchiyama Y and Nagata S (1998) Caspase-independent cell killing by Fas-associated protein with death domain. J. Cell. Biol. 143: 1353-1360 